\title{
Doping-Induced Electron Transfer at Organic/Oxide Interfaces: Direct Evidence from Infrared Spectroscopy
}

\author{
L. Schöttner," S. Erker," R. Schlesinger, N. Koch, A. Nefedov, O. T. Hofmann, and C. Wöll* \\ Cite This: J. Phys. Chem. C 2020, 124, 4511-4516 \\ Read Online
}

ABSTRACT: Charge transfer at organic/inorganic interfaces critically influences the properties of molecular adlayers. Although for metals such charge transfers are well documented by experimental and theoretical results, in the case of semiconductors, clear and direct evidence for a transfer of electrons or holes from oxides with their typically high ionization energy is missing. Here, we present data from infrared reflection-absorption spectroscopy demonstrating that despite a high ionization energy, electrons are transferred from $\mathrm{ZnO}$ into a prototype strong molecular electron acceptor, hexafluoro-tetracyano-naphthoquinodimethane ( $\mathrm{F}_{6}$-TCNNQ). Because there are no previous studies of this type, the interpretation of the pronounced

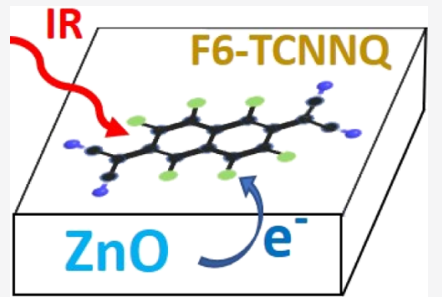
vibrational red shifts observed in the experiment was aided by a thorough theoretical analysis using density functional theory. The calculations reveal that two mechanisms govern the pronounced vibrational band shifts of the adsorbed molecules: electron transfer into unoccupied molecular levels of the organic acceptor and also the bonding between the surface $\mathrm{Zn}$ atoms and the peripheral cyano groups. These combined experimental data and the theoretical analysis provide the so-far missing evidence of interfacial electron transfer from high ionization energy inorganic semiconductors to molecular acceptors and indicates that $\mathrm{n}$-doping of $\mathrm{ZnO}$ plays a crucial role.

\section{INTRODUCTION}

Organic/inorganic interfaces attract substantial interest because of the prospect to exploit electronic and optical effects occurring at such heterojunctions for applications in optics, electronics, energy storage and conversion, catalysis, and sensing. ${ }^{1}$ The often unusual behavior at such interfaces results from combining two materials with rather different properties, for example, electronic structure, dielectric constants, and polarizabilities. In general, depending on the relative energies of the rather localized molecular orbital levels and the more dispersive electronic bands in the inorganic semiconductor, interfacial charge transfer can take place, leading to the formation of electric fields at these hybrid interfaces. ${ }^{2}$ A unique prospect for engineering the properties of devices exploiting these effects arises from the possibility to tune these charge density rearrangements by doping, in particular, that of the inorganic part.

However, even for an easy-to-realize class of organic/ inorganic interfaces-organic adlayers on inorganic substrates-the nature of the charge transfer remains elusive. An interesting wide band gap semiconductor for such heterointerfaces is the metal oxide $\mathrm{ZnO}$, and a number of studies have been reported recently in this context. ${ }^{3-6}$ Here, a large work function increase was observed for the adsorption of strong electron acceptors, such as tetrafluoro-tetracyano-quinodimethane $\left(\mathrm{F}_{4}\right.$-TCNQ). ${ }^{3}$ A straightforward explanation for this observation would be a substantial charge transfer from the $\mathrm{ZnO}$ substrate to the adsorbed electron acceptor, similar to the situation on coinage metals. However, evidence for such a large transfer, for example, in the form of a filled lowest unoccupied molecular orbital (LUMO) appearing in the $\mathrm{ZnO}$ band gap (as indicated in Figure 1), is missing from ultraviolet photoelectron spectroscopy (UPS) experiments. ${ }^{3}$ In theory, for a moderately doped semiconductor, band-bending effects could reduce the amount of transferred charge up to the point where the molecules would be essentially uncharged. ${ }^{3,7}$

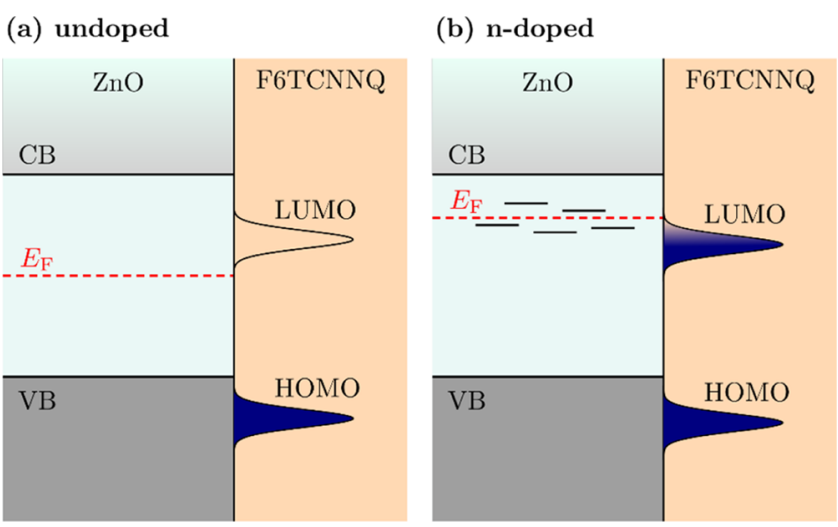

Figure 1. Schematic energy level diagrams of electron acceptor molecules on (a) an undoped semiconductor and (b) an n-doped semiconductor.

Received: September 16, 2019

Revised: January 27, 2020

Published: January 29, 2020 

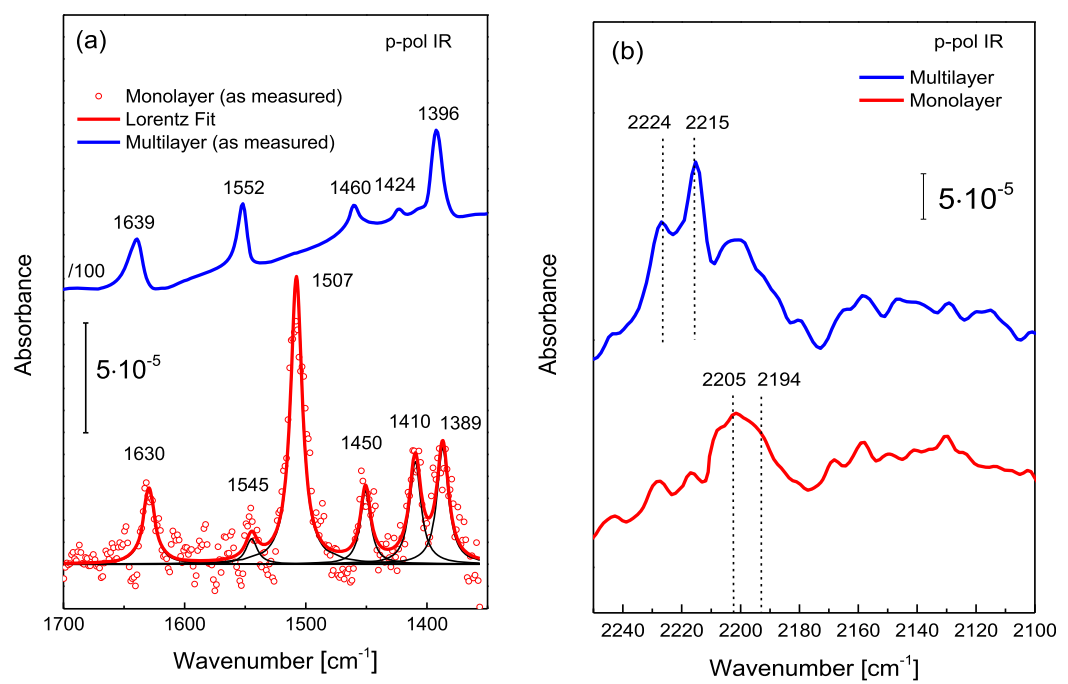

Figure 2. Experimental IRRAS spectra of $\mathrm{F}_{6}$-TCNNQ on the nominally undoped $\mathrm{ZnO}(10 \overline{1} 0$ ) measured with p-polarized light: (a) $\mathrm{C}=\mathrm{C} / \mathrm{C}-\mathrm{C}$ region, the intensity of the multilayer data are scaled by a factor of 100 and $(\mathrm{b}) \mathrm{C} \equiv \mathrm{N}$ region.

In this contribution, we challenge the interpretation of basically neutral molecular adsorbates. Our data from infrared reflection-absorption spectroscopy (IRRAS) directly reveal that hexafluoro-tetracyano-naphthoquinodimethane $\left(\mathrm{F}_{6}\right.$ TCNNQ), a common p-dopant for organic semiconductors, ${ }^{8-10}$ becomes strongly negatively charged upon adsorption.

For this purpose, $\mathrm{F}_{6}$-TCNNQ was deposited on a structurally well-defined $\mathrm{ZnO}(10 \overline{1} 0)$ surface. IRRAS allows detecting charge transfer across the interface by measuring adsorptioninduced changes of molecular vibrational frequencies. For metal substrates, IRRAS has already been applied successfully to evidence interfacial charge transfer by detecting frequency shifts of vibrations in molecular adsorbates. ${ }^{1-14}$ For PTCDA, another common, planar aromatic compound, adsorbed on $\operatorname{Ag}(111)$, the changes in frequency were found to amount to $10-30 \mathrm{~cm}^{-1} .11$ However, similar studies for molecular adsorbates on wide band gap inorganic semiconductors have not yet been reported. This is mainly due to severe challenges in measuring infrared (IR) spectra in reflection for nonmetallic substrates. Only more recently, these problems have been overcome by a number of groups (for a review, see ref 15 ).

\section{EXPERIMENTAL SECTION}

The experiments were conducted in a state-of-the-art ultrahigh vacuum (UHV) multichamber system (Prevac). ${ }^{15}$ The apparatus allows performing IR spectroscopy in combination with other surface sensitive techniques such as X-ray-photoelectron spectroscopy (XPS) and low-energy electron diffraction (LEED). The IR spectra were obtained in the reflectionabsorption mode (IRRAS) using a Fourier-transform (FT)-IR spectrometer (Bruker VERTEX 80v) directly coupled to the UHV chamber via differentially pumped $\mathrm{KBr}$-windows. The base pressure of the entire chamber is in the $10^{-10} \mathrm{mbar}$ range.

As substrates, one nominally undoped $\mathrm{ZnO}(10 \overline{1} 0)$ single crystal (CrysTec) and two Ga-doped $\mathrm{ZnO}(10 \overline{10})$ thin films grown on the bulk $\mathrm{ZnO}(10 \overline{1} 0)$ single crystal by molecular beam epitaxy were used. In the latter case, the charge carrier density was determined by analyzing far-IR transmission spectra in the context of the Drude model, ${ }^{16}$ yielding n-doping levels of $7.8 \times$ $10^{19}$ and $2.9 \times 10^{20} \mathrm{~cm}^{-3}$, respectively.
The substrates were cleaned by repeated cycles of $\mathrm{Ar}^{+}$ sputtering $\left(1 \times 10^{-6} \mathrm{mbar}, 10 \mathrm{~min}\right)$ and annealing for $10 \mathrm{~min}$ at $800 \mathrm{~K}$ in a case of the undoped sample and at $700 \mathrm{~K}$ for Gadoped $\mathrm{ZnO}$. As a final step, the samples were annealed in an oxygen atmosphere $\left(1 \times 10^{-6}\right.$ mbar $)$ at 840 and $700 \mathrm{~K}$ for undoped and Ga-doped samples, respectively. The lower annealing temperature was applied for Ga-doped $\mathrm{ZnO}$ to keep the doping level unchanged. The cleanliness and structural quality of the substrate surface were monitored by XPS and LEED.

Deposition of $\mathrm{F}_{6}$-TCNNQ (Novaled) on $\mathrm{ZnO}(10 \overline{1} 0$ ) was carried out by directing the flux of an effusion cell toward the substrate. Deposition rates were determined using a quartz crystal microbalance. Based on the determined deposition rate, we estimate that the coverage of the monolayers used to record the data shown in Figure 2 amounted to $0.7 \pm 0.3$ monolayer, that is slightly less than a full monolayer (one monolayer corresponds to a surface fully covered by flat-lying molecules). For larger coverage (i.e., longer deposition times), bilayer formation was observed in the IR data. According to the calculated adsorption geometry (see Figure SI1, Supporting Information), monolayer coverage corresponds to $\sim 4 \times 10^{13}$ molecule per $\mathrm{cm}^{2}$. This value is in good agreement with the estimates made by Katayama ${ }^{17}$ for $\mathrm{F}_{4}$-TCNQ considering the larger size of the $\mathrm{F}_{6}$-TCNNQ molecule. For the $\mathrm{F}_{6}$-TCNNQ multilayer, the deposition time was 10 times longer; therefore, we estimate a film thickness of $\sim 70 \AA$.

All IRRAS experiments were carried out at room temperature. An internal polarizer module allowed to record IR spectra with sand p-polarized light. All IR spectra shown here are difference spectra obtained by subtracting a spectrum recorded for the clean $\mathrm{ZnO}$ substrate before $\mathrm{F}_{6}$-TCNNQ deposition from that measured after molecule deposition. Note that in contrast to metal substrates, the bands in the IRRAS spectra can have different signs, positive or negative. For a detailed discussion, see the previous literature. ${ }^{15,18}$ The angle of incidence of the IR light was set to $80^{\circ}$ with respect to the surface normal because according to previous spectrum simulations for adsorbates on $\mathrm{ZnO},{ }^{18}$ the p-polarized signal adopts a maximum at $80-85^{\circ}$, while for s-polarized light, the dependence on $\Theta$ is only weak. 
Table 1. Experimental and Theoretical Results for $\mathrm{F}_{6}$-TCNNQ

\begin{tabular}{|c|c|c|c|c|c|c|}
\hline symmetry/vibration & $\begin{array}{l}\text { experiment, } \\
\text { multilayer, } \mathrm{cm}^{-1}\end{array}$ & $\begin{array}{c}\text { experiment, } \\
\text { monolayer, } \mathrm{cm}^{-1}\end{array}$ & $\begin{array}{l}\text { theory }{ }^{a} \text {, gas phase, } \\
\text { neutral, } \mathrm{cm}^{-1}\end{array}$ & $\begin{array}{l}\text { theory }^{a} \text {, gas phase, } \\
\text { charged, } \mathrm{cm}^{-1}\end{array}$ & $\begin{array}{l}\text { theory }^{a} \text {, surface, } \\
\text { neutral, } \mathrm{cm}^{-1}\end{array}$ & $\begin{array}{l}\text { theory }^{a} \text {, surface, } \\
\text { charged, } \mathrm{cm}^{-1}\end{array}$ \\
\hline \multicolumn{7}{|c|}{$\mathrm{C}-\mathrm{N}$ Vibrations } \\
\hline $\mathrm{b}_{\mathrm{u}} \nu_{49}$ & 2224 & 2205 & 2248 & 2222 & 2229 & 2202 \\
\hline$a_{\mathrm{g}} \nu_{1}$ & 2224 & 2205 & 2246 & 2223 & 2219 & 2196 \\
\hline $\mathrm{b}_{\mathrm{u}} \nu_{50}$ & 2215 & 2194 & 2221 & 2189 & 2197 & 2165 \\
\hline$a_{\mathrm{g}} \nu_{2}$ & 2215 & 2194 & 2221 & 2189 & 2191 & 2158 \\
\hline \multicolumn{7}{|c|}{$\mathrm{C}-\mathrm{C}$ Vibrations } \\
\hline $\mathrm{b}_{\mathrm{u}} \nu_{51}$ & 1639 & 1630 & 1638 & 1629 & 1632 & 1622 \\
\hline$a_{\mathrm{g}} \nu_{4}$ & 1552 & 1545 & 1552 & 1551 & 1551 & 1550 \\
\hline $\mathrm{b}_{\mathrm{u}} \nu_{52}$ & 1552 & 1507 & 1552 & 1508 & 1567 & 1522 \\
\hline $\mathrm{b}_{\mathrm{u}} \nu_{53}$ & 1460 & 1450 & 1470 & 1445 & 1490 & 1465 \\
\hline $\mathrm{b}_{\mathrm{u}} \nu_{54}$ & 1424 & 1410 & 1431 & 1412 & 1434 & 1415 \\
\hline $\mathrm{b}_{\mathrm{u}} \nu_{55}$ & 1396 & 1389 & 1402 & 1379 & 1420 & 1397 \\
\hline
\end{tabular}

\section{COMPUTATIONAL DETAILS}

As mentioned in the introduction, obtaining a reliable prediction of the charge state of the adsorbed molecule via semi-local density functional theory (DFT) is challenging. This is because the LUMO is spuriously below the valence band of $\mathrm{ZnO}$, which yields superficial charge transfer, independent of the substrate's actual Fermi level. In principle, this problem can be solved using more sophisticated methods, such as DFT $+U^{19}$ or hybrid DFT. ${ }^{20}$ However, in either case, the amount of charge transfer depends sensitively on the parameter used to account for the Coulomb on-site energy (the Hubbard $U$ or the amount of exact exchange) and on the precise doping concentration (and its nature), ${ }^{19,20}$ which still prevents a stand-alone computational prediction. Therefore, we pursue a different approach here: rather than calculating the charge state directly, the aim of the calculations is to predict the shift of vibrational frequencies as a consequence of (a) charging of the $\mathrm{F}_{6}$-TCNNQ molecule and (b) binding of the molecule to the surface. We do this separately by calculating the shift due to charging for a free molecule in the gas phase and by calculating the vibrational frequencies for the molecule on the surface (where, in our calculations, we assume a charge transfer of approximately one electron). Assuming that these two effects are independent and additive, we can combine them to estimate the vibrational shift for arbitrarily charged molecules on the surface, as explained in more detail in the Supporting Information. Comparing the frequencies observed in the IRRAS experiment to the computed values then allows us to extract the factual charge state of the molecule on the surface.

All computed vibrational modes presented here were obtained by DFT calculations using the FHI-aims program package. $^{21}$ The Perdew-Burke-Ernzerhof $(\mathrm{PBE})^{22}$ density functional was used and van der Waals forces were included with the vdW-TS scheme, ${ }^{23}$ using the appropriate parameters for the $\mathrm{ZnO}$ substrate. $^{24}$ The vibrational modes were computed numerically via the finite displacement method using a displacement amplitude of $0.005 \AA$. Initial geometries were optimized until residual forces were smaller than $10^{-4} \mathrm{eV} / \AA ̊$ with "tight" basis sets and numerical settings.

Calculations for the free molecule were performed with open boundary conditions. The electronic structure of the $\mathrm{F}_{6}$ TCNNQ/ZnO interface was determined using a periodic slab approach. The $\mathrm{ZnO}$ substrate was modeled using a five doublelayer slab cell. For geometry optimization, the bottom three double-layers were fixed at their bulk positions and the top two layers were allowed to relax. For the vibrational analysis, we restrict the discussion to vibrations of the molecule within the harmonic approximation. By keeping the substrate fixed, we omit changes in pure surface and pure bulk modes. To avoid artifacts arising from artificial surface states located at the bottom side of the slab, the bottom layer was passivated with hydrogen atoms. Calculations for the primitive surface unit cell are performed with a $16 \times 24 \times 1 \mathrm{k}$-grid and scaled accordingly for larger cells. The $4 \times 4$ supercell including the molecule was chosen such that there is no significant intermolecular interaction between the adjacent molecular adsorbates when employing periodic boundary conditions.

The adsorption of the $\mathrm{F}_{6}$-TCNNQ molecules on the $\mathrm{ZnO}(10 \overline{10})$ surface is governed by weak binding of the protruding cyano groups to the surface $\mathrm{Zn}$ atoms, which act as docking sites, leading to a planar molecular adsorption geometry. Two different azimuthal orientations were considered: oriented along the rills of the mixed-terminated $\mathrm{ZnO}$ surface (i.e., along the $\mathrm{ZnO}[1 \overline{2} 10]$ direction) and oriented perpendicular to the rills (the $\mathrm{ZnO}[0001]$ direction). The orientation perpendicular to the rills is energetically more favorable by $33 \mathrm{meV}$ and was therefore used for the vibrational analysis in this work.

Vibrational frequencies usually strongly depend not only on the physics of the system but also on the density functional and the basis set used in the DFT calculations. Because of the systematic nature of the deviations, it is customary to apply uniform multiplicative scale factors to correct the computed vibrational frequencies. ${ }^{25-27}$ The scale factor used here was determined from experimental and theoretical data for $\mathrm{F}_{4^{-}}$ TCNQ. $^{28-30}$ This procedure yields scale factors of 1.0095 for the $\mathrm{C} \equiv \mathrm{N}$ and of 1.0198 for the $\mathrm{C}=\mathrm{C} / \mathrm{C}-\mathrm{C}$ vibrational frequencies. (More details on the computational methods including how we obtained the scale factors can be found in the Supporting Information, Chapter 4.)

\section{RESULTS AND DISCUSSION}

To determine the charge state of the molecules in direct contact with the substrate, it is useful to first characterize a molecular multilayer, where the molecules are clearly charge neutral. This provides a baseline for comparing the vibrational signatures of the experiment and theory, allowing us to analyze shifts due to charging and binding to the surface afterward. In the following, we focus on the results for nominally undoped $\mathrm{ZnO}(10 \overline{1} 0)$, which is actually unintentionally n-type doped; ${ }^{31,32}$ and the results are qualitatively equivalent to those obtained for highly 
doped samples. It is necessary to mention that the IRRAS data recorded for $\mathrm{F}_{6}$-TCNNQ multilayers adsorbed on the Ga-doped $\mathrm{ZnO}$ samples look the same as for those adsorbed on the nominally undoped $\mathrm{ZnO}$ sample. Moreover, we measured thick (1000 A) multilayers of $\mathrm{F}_{6}$-TCNNQ deposited on the oxidized $\mathrm{Si}$ wafer. The multilayer spectra recorded for these different substrates are virtually identical. Moreover, during coveragedependent experiments, it was found that after completion of the monolayer, we immediately start to observe the features typical for the multilayer. This observation allows us to conclude that the charge transfer is limited strictly to the first adsorbed layer and that molecules further away are only affected very weakly by the presence of the $\mathrm{ZnO}$ substrate.

The inspection of the IRRAS data from multilayer $\mathrm{F}_{6^{-}}$ TCNNQ (Figure 2, top curves) reveals several well-defined, sharp vibrational bands. In the frequency regime below 1700 $\mathrm{cm}^{-1}$ ( $\mathrm{C}=\mathrm{C} / \mathrm{C}-\mathrm{C}$ region), four strong bands are seen in the experimental multilayer data, centered at 1639, 1552, 1460, and $1396 \mathrm{~cm}^{-1}$. In addition, a small peak is seen at $1424 \mathrm{~cm}^{-1}$. For their assignment, we rely on the comparison to the corresponding data for a structurally related molecule, $\mathrm{F}_{4^{-}}$ $\mathrm{TCNQ}^{28-30}$ and to the results of DFT calculations for $\mathrm{F}_{6}$ TCNNQ in the gas phase (see Table 1).

Overall, the agreement between the positions of the bands in the experimental multilayer IR spectra and the DFT results for the free molecule is very good. Note that the small deviation between the experiment and theory is similar to that reported in previous work for $\mathrm{F}_{4}$-TCNQ multilayers. ${ }^{29}$ On the basis of this comparison, in Table 1 (column "theory, gas phase, neutral"), we assign the four strong bands to the $\mathrm{C}-\mathrm{C}$ vibrations $\mathrm{b}_{\mathrm{u}} \nu_{51}$, $\mathrm{b}_{\mathrm{u}} \nu_{52}, \mathrm{~b}_{\mathrm{u}} \nu_{53}$, and $\mathrm{b}_{\mathrm{u}} \nu_{55}$ and the weak feature to $\mathrm{b}_{\mathrm{u}} \nu_{54}$. In the $\mathrm{C} \equiv$ $\mathrm{N}$ stretch regime, we observe four features at 2224, 2215, 2205, and $2194 \mathrm{~cm}^{-1}$ (Figure 2b, top curve). We assign the band at $2224 \mathrm{~cm}^{-1}$ to the degenerate $b_{\mathrm{u}} \nu_{49} / a_{g} \nu_{1}$ vibrations and the band at $2215 \mathrm{~cm}^{-1}$ to the degenerate $b_{u} \nu_{50} / a_{g} \nu_{2}$ vibrations (see Table 1 ). This assignment is in analogy with that reported for $F_{4}$ TCNQ multilayers. ${ }^{29}$ Two features at lower wavenumbers are ascribed to signals still originating from the monolayer, see below. We would like to note that for $\mathrm{F}_{4}-\mathrm{TCNQ} /$ (co)polymer blends, an additional strong band at $2169 \mathrm{~cm}^{-1}$ has been found. ${ }^{29}$ We were unable to observe such vibration band in our data, and all features seen in Figure $2 b$ in the range 2180-2100 $\mathrm{cm}^{-1}$ are below the noise level.

Unfortunately, the bulk crystal structure of $\mathrm{F}_{6}$-TCNNQ is unknown. Also, in the case of thin films, Duva et al. ${ }^{33}$ were unable to deduce structural parameters because out-of-plane diffraction peaks for $\mathrm{F}_{6}$-TCNNQ films could not be detected. However, we note that all vibrational modes discussed above have their transition dipole moments oriented within the molecular plane of $\mathrm{F}_{6}$-TCNNQ. Note that the experimental $\mathrm{p}$ polarized multilayer data (Figure 2 ) do not reveal any vibrational bands with a negative signal (for a discussion of the interplay between orientation of transition dipole moments, the polarization of the incident IR-light, and the sign of vibrational bands in IRRAS at dielectric surfaces, see the Supporting Information). This observation implies that all $\mathrm{F}_{6}$-TCNNQ molecules must be orientated with their molecular plane parallel to the $\mathrm{ZnO}$ substrate, see Figure 3. IRRAS data recorded with s-polarization (see Figure SI11 in the Supporting Information) are fully consistent with parallel stacking in multilayers of this aromatic compound.

For the $\mathrm{F}_{6}$-TCNNQ monolayer on $\mathrm{ZnO}(10 \overline{1} 0)$, the IRRAS data reveal pronounced differences compared to the multilayer

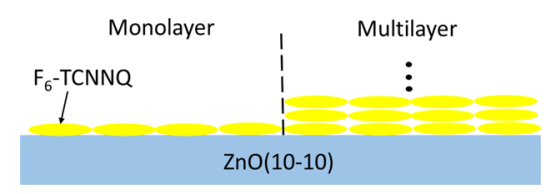

Figure 3. Orientation of $\mathrm{F}_{6}$-TCNNQ in the monolayer (left) and multilayer (right) regimes. The polarization dependence of the IR-data indicates an orientation of the molecular plane parallel to the substrate.

spectra (see Figure 2a, bottom curve). For the monolayer, the most pronounced peak is that at $1507 \mathrm{~cm}^{-1}$. Lower intensity bands are observed at 1630,1450,1410, and $1389 \mathrm{~cm}^{-1}$, and a small feature is observed at $1545 \mathrm{~cm}^{-1}$.

In case of a neutral $\mathrm{F}_{6}$-TCNNQ adsorbate, the DFT results predict a small blue shift $\left(15 \mathrm{~cm}^{-1}\right)$ of the strong $b_{\mathrm{u}} \nu_{52}$ band at $1552 \mathrm{~cm}^{-1}$ relative to the molecule in the gas phase because of the bonding of the molecule to the surface. ${ }^{34}$ Because experimentally, the peak actually shifts in the opposite direction, we conclude that the presence of neutral $\mathrm{F}_{6}$-TCNNQ molecules at the interface is unlikely. Conversely, for a negatively charged molecular species, the calculations predict a red shift of $30 \mathrm{~cm}^{-1}$ for the $\mathrm{b}_{\mathrm{u}} \nu_{52}$ band (see Figure SI5 in the Supporting Information), in agreement with the experimentally observed shift of $45 \mathrm{~cm}^{-1}$. Such a substantial lowering of frequencies is typical for electron transfer to this organic acceptor. ${ }^{30}$ In line with this hypothesis, the second sharp band for the monolayer observed at $1450 \mathrm{~cm}^{-1}$ can be assigned to the $b_{u} \nu_{53}$ band $(1460$ $\mathrm{cm}^{-1}$ in the multilayer), that is, a red shift of $10 \mathrm{~cm}^{-1}$ is observed for the monolayer. Also, in this case, the DFT results for the neutral species predict a small blue shift, whereas for the charged species, a small red shift is expected $\left(5 \mathrm{~cm}^{-1}\right)$. Inspection of Table 1 confirms that the other vibrations also agree well with the theoretical results for a $\mathrm{F}_{6}$-TCNNQ anion at the interface with $\mathrm{ZnO}$.

Assuming a charge transfer of 1 electron/molecule for the full monolayer, $\sim 10^{14}$ electrons for the transfer from the substrate to $\mathrm{F}_{6}$-TCNNQ are needed. This value is absolutely reasonable for the $\mathrm{n}$-doping levels present in the substrate.

In the $\mathrm{C} \equiv \mathrm{N}$-stretch region, the experimental monolayer data reveal only one rather broad feature $\left(2212-2188 \mathrm{~cm}^{-1}\right.$ ) centered at $2205 \mathrm{~cm}^{-1}$ (Figure $2 \mathrm{~b}$, bottom curve). The center of this band is red-shifted by $10-20 \mathrm{~cm}^{-1}$ with respect to the strongest multilayer peaks at $2215 \mathrm{~cm}^{-1}$ and $2224 \mathrm{~cm}^{-1}$. Although the lower intensity of the $\mathrm{C} \equiv \mathrm{N}$ bands in the experimental data prohibits a more detailed analysis, the red shift in the measured monolayer data is consistent with the theoretical results for the negatively charged species, where for all $\mathrm{C} \equiv \mathrm{N}$ stretch vibrations, red-shifts between 40 and $60 \mathrm{~cm}^{-1}$ are found.

Analysis of the experimental data confirms the plane of the $\mathrm{F}_{6}$ TCNNQ monolayer to be orientated parallel to the substrate, as also indicated by the theoretical results.

Overall, our findings for the nominally undoped $\mathrm{ZnO}$ substrate clearly show that the adsorbate is negatively charged, in variance to the naive expectation depicted in Figure $1 \mathrm{~b}$. Importantly, this is in agreement with the observation that the measured band bending for semiconductor/organic interfaces is significantly smaller than that required to obtain charge-neutral adsorbates. 3,35 Notably, although theory suggests that the amount of charge transfer should be dependent on the doping concentration, ${ }^{3,20}$ experiments for more strongly doped samples showed no difference to the nominally undoped sample. 
As a final remark, we note that our experimental and theoretical findings cannot reconcile the apparent absence of filled molecular states in previous ultraviolet photoelectron spectroscopy experiments. Possible origins include strong charge localization in the molecular layer, which could shift the filled states far below $E_{\mathrm{F}}{ }^{36,37}$ or a pronounced hybridization of the surface states with the molecular orbitals, which could also lead to masking of ebbing molecular states by the $\mathrm{ZnO}$ valence band. However, a conclusive answer would require significant improvements over state-of-the-art theory, which is outside the scope of the present contribution.

\section{CONCLUSIONS}

The experimental IRRAS data obtained for $\mathrm{F}_{6}$-TCNNQ molecules deposited on a $\mathrm{ZnO}(10 \overline{1} 0)$ surface reveal the formation of multilayers with the molecular plane orientated strictly parallel to the substrate. In the $\mathrm{C} \equiv \mathrm{N}$ region for the $\mathrm{F}_{6^{-}}$ TCNNQ monolayer, two sharp vibrational bands are observed, with pronounced red shifts relative to the multilayer. As confirmed by DFT results, these shifts are inconsistent with a neutral adsorbate. Instead, from the good agreement with theoretical results obtained for a molecular anion, we conclude that $\mathrm{F}_{6}$-TCNNQ molecules in the monolayer are negatively charged. This information provides the so-far missing evidence of interfacial electron transfer from high ionization energy inorganic semiconductors to molecular acceptors. In this case, the transfer is made possible by $\mathrm{n}$-doping. Consequently, the comprehensive understanding of charge rearrangements at inorganic/organic interfaces is substantially ameliorated and can now be exploited to further tailor such interfaces for advanced applications.

\section{ASSOCIATED CONTENT}

\section{(s) Supporting Information}

The Supporting Information is available free of charge at https://pubs.acs.org/doi/10.1021/acs.jpcc.9b08768.

Top and side view of the theoretically found lowest energy local adsorption site of $\mathrm{F}_{6}$-TCNNQ on the $\mathrm{ZnO}$ surface; MODOS and orbital population analysis of $\mathrm{F}_{6}$-TCNNQ on $\mathrm{ZnO}$; most relevant molecular orbitals for charge transfer upon adsorption of $\mathrm{F}_{6}$-TCNNQ on $\mathrm{ZnO}$, two most prominent features that appear in gas-phase calculations; depiction of $\mathrm{C}=\mathrm{C} / \mathrm{C}-\mathrm{C}$ and $\mathrm{C} \equiv \mathrm{N}$ frequency shifts of flat-laying $\mathrm{F}_{6}$-TCNNQ; theoretical results of $\mathrm{C}-\mathrm{C} / \mathrm{C}=\mathrm{C}$ stretching vibrations; theoretical results of $\mathrm{C} \equiv \mathrm{N}$ stretching vibrations; chemical structure and double bond locations within the molecule; bond lengths of all bonds within the $\mathrm{F}_{6}$-TCNNQ molecule; and experimental IRRAS spectra of $\mathrm{F}_{6}$-TCNNQ measured with $\mathrm{p}$ - and s-polarized light (PDF)

\section{AUTHOR INFORMATION}

\section{Corresponding Author}

C. Wöll - Karlsruhe Institute of Technology, Institute of Functional Interfaces, 76344 Eggenstein-Leopoldshafen, Germany; ○ orcid.org/0000-0003-1078-3304; Email: christof.woell@ kit.edu

\section{Authors}

L. Schöttner - Karlsruhe Institute of Technology, Institute of Functional Interfaces, 76344 Eggenstein-Leopoldshafen, Germany
S. Erker - Graz University of Technology, 8010 Graz, Austria

R. Schlesinger - Humboldt Universität zu Berlin, Institut für Physik \& IRIS Adlershof, 12489 Berlin, Germany

N. Koch - Humboldt Universität zu Berlin, Institut für Physik \& IRIS Adlershof, 12489 Berlin, Germany; 잉o․org/00000002-6042-6447

A. Nefedov - Karlsruhe Institute of Technology, Institute of Functional Interfaces, 76344 Eggenstein-Leopoldshafen, Germany; 이이이.org/0000-0003-2771-6386

O. T. Hofmann - Graz University of Technology, 8010 Graz, Austria; (1) orcid.org/0000-0002-2120-3259

Complete contact information is available at:

https://pubs.acs.org/10.1021/acs.jpcc.9b08768

\section{Author Contributions}

"L.S. and S.E. contributed equally to this work.

\section{Notes}

The authors declare no competing financial interest.

\section{ACKNOWLEDGMENTS}

Helmholtz Research School "Energy-related catalysis" is gratefully acknowledged for providing financial support and the donation of a PhD-Scholarship to L.S. S.E. and O.H. acknowledge funding from the Austrian Science Fund (FWF P27868-N36). The computational results presented in this article were obtained using the resources of the Vienna Scientific Cluster (VSC). R.S. and N.K. acknowledge support by the Deutsche Forschungsgemeinschaft (DFG)-project number 182087777-SFB 951. We thank S. Sadofev and T. Meisel for providing the $\mathrm{Ga}$-doped $\mathrm{ZnO}$ samples.

\section{REFERENCES}

(1) Delville, M. H.; Taubert, A. Hybrid Organic-Inorganic Interfaces: towards Advanced Functional Materials, B and 1; John Wiley \& Sons: Weinheim, Germany, 2018; p 1016.

(2) Hörmann, U.; Zeiske, S.; Piersimoni, F.; Hoffmann, L.; Schlesinger, R.; Koch, N.; Riedl, T.; Andrienko, D.; Neher, D. Stark effect of hybrid charge transfer states at planar $\mathrm{ZnO}$ /organic interfaces. Phys. Rev. B 2018, 98, 155312 .

(3) Schlesinger, R.; Xu, Y.; Hofmann, O. T.; Winkler, S.; Frisch, J.; Niederhausen, J.; Vollmer, A.; Blumstengel, S.; Henneberger, F.; Rinke, $\mathrm{P}$. Controlling the work function of $\mathrm{ZnO}$ and the energy-level alignment at the interface to organic semiconductors with a molecular electron acceptor. Phys. Rev. B 2013, 87, 155311.

(4) Pal, S.; Maiti, S.; Maiti, U. N.; Chattopadhyay, K. K. ZnO-(Cu/ $\mathrm{Ag}$ ) TCNQ heterostructure network over flexible platform for enhanced cold cathode application. Nanotechnology 2016, 27, 265601.

(5) Liu, H.; Cui, S.; Guo, Y.; Li, Y.; Huang, C.; Zuo, Z.; Yin, X.; Song, Y.; Zhu, D. Fabrication of large-area hybrid nanowires arrays as novel field emitters. J. Mater. Chem. 2009, 19, 1031-1036.

(6) Hosaka, H.; Meguro, K. The solvent effect on the charge-transfer adsorption of TCNQ onto metal oxides and silica. Colloid Polym. Sci. 1974, 252, 322-326.

(7) Xu, Y.; Hofmann, O.; Schlesinger, R.; Winkler, S.; Frisch, J.; Niederhausen, J.; Vollmer, A.; Blumstengel, S.; Henneberger, F.; Koch, N.; Rinke, P.; Scheffler, M. Space-Charge Transfer in Hybrid InorganicOrganic Systems. Phys. Rev. Lett. 2013, 111, 226802.

(8) Kleemann, H.; Schuenemann, C.; Zakhidov, A. A.; Riede, M.; Lüssem, B.; Leo, K. Structural phase transition in pentacene caused by molecular doping and its effect on charge carrier mobility. Org. Electron. 2012, 13, 58-65.

(9) Weichsel, C.; Burtone, L.; Reineke, S.; Hintschich, S. I.; Gather, M. C.; Leo, K.; Lüssem, B. Storage of charge carriers on emitter molecules in organic light-emitting diodes. Phys. Rev. B 2012, 86, 075204. 
(10) Tietze, M. L.; Burtone, L.; Riede, M.; Lüssem, B.; Leo, K. Fermi level shift and doping efficiency in p-doped small molecule organic semiconductors: a photoelectron spectroscopy and theoretical study. Phys. Rev. B 2012, 86, 035320.

(11) Zaitsev, N. L.; Jakob, P.; Tonner, R. Structure and vibrational properties of the PTCDA/Ag (111) interface: bilayer versus monolayer. J. Phys.: Condens. Matter 2018, 30, 354001.

(12) Bröker, B.; Hofmann, O. T.; Rangger, G. M.; Frank, P.; Blum, R.P.; Rieger, R.; Venema, L.; Vollmer, A.; Müllen, K.; Rabe, J. P.; Winkler, A.; Rudolf, P.; Zojer, E.; Koch, N. Density-Dependent Reorientation and Rehybridization of Chemisorbed Conjugated Molecules for Controlling Interface Electronic Structure. Phys. Rev. Lett. 2010, 104, 246805.

(13) Skibbe, O.; Binder, M.; Otto, A.; Pucci, A. Electronic contributions to infrared spectra of adsorbate molecules on metal surfaces: Ethene on $\mathrm{Cu}(111)$. J. Chem. Phys. 2008, 128, 194703.

(14) Beck, S.; Gerbert, D.; Glaser, T.; Pucci, A. Charge Transfer at Organic/Inorganic Interfaces and the Formation of Space Charge Regions Studied with Infrared Light. J. Phys. Chem. C 2015, 119, $12545-12550$.

(15) Yang, C.; Wöll, C. IR spectroscopy applied to metal oxide surfaces: adsorbate vibrations and beyond. Adv. Phys.: X 2017, 2, 373408.

(16) Manciu, F. S.; Manciu, M.; Durrer, W. G.; Salazar, J. G.; Lee, K. H.; Bennet, K. E. A Drude model analysis of conductivity and free carriers in boron-doped diamond films and investigations of their internal stress and strain. J. Mater. Sci. 2014, 49, 5782-5789.

(17) Katayama, T.; Mukai, K.; Yoshimoto, S.; Yoshinobu, J. Thermally Activated Transformation from a Charge-Transfer State to a Rehybridizied State of Tetrafluoro-tetracyanoquinodimethane on $\mathrm{Cu}(100)$. J. Phys. Chem. Lett. 2010, 1, 2917-2921.

(18) Buchholz, M.; Weidler, P. G.; Bebensee, F.; Nefedov, A.; Wöll, C. Carbon dioxide adsorption on a $\mathrm{ZnO}(10-10)$ substrate studied by infrared reflection absorption spectroscopy. Phys. Chem. Chem. Phys. 2014, 16, 1672.

(19) Gruenewald, M.; Schirra, L. K.; Winget, P.; Kozlik, M.; Ndione, P. F.; Sigdel, A. K.; Berry, J. J.; Forker, R.; Brédas, J.-L.; Fritz, T.; Monti, O. L. A. Integer Charge Transfer and Hybridization at an Organic Semiconductor/Conductive Oxide Interface. J. Phys. Chem. C 2015, $119,4865$.

(20) Erker, S.; Hofmann, O. T. Fractional and Integer Charge Transfer at Semiconductor/Organic Interfaces: The Role of Hybridization and Metallicity. J. Phys. Chem. Lett. 2019, 10, 848-854.

(21) Blum, V.; Gehrke, R.; Hanke, F.; Havu, P.; Havu, V.; Ren, X.; Reuter, K.; Scheffler, M. Ab initio molecular simulations with numeric atom-centered orbitals. Comput. Phys. Commun. 2009, 180, 21752196.

(22) Perdew, J. P.; Burke, K.; Ernzerhof, M. Generalized gradient approximation made simple. Phys. Rev. Lett. 1996, 77, 3865.

(23) Tkatchenko, A.; Scheffler, M. Accurate molecular van der Waals interactions from ground-state electron density and free-atom reference data. Phys. Rev. Lett. 2009, 102, 073005.

(24) Hofmann, O. T.; Deinert, J.-C.; Xu, Y.; Rinke, P.; Stähler, J.; Wolf, M.; Scheffler, M. Large work function reduction by adsorption of a molecule with a negative electron affinity: Pyridine on $\mathrm{ZnO}(10-10)$. J. Chem. Phys. 2013, 139, 174701.

(25) Laury, M. L.; Carlson, M. J.; Wilson, A. K. Vibrational frequency scale factors for density functional theory and the polarization consistent basis sets. J. Comput. Chem. 2012, 33, 2380-2387.

(26) Merrick, J. P.; Moran, D.; Radom, L. An Evaluation of Harmonic Vibrational Frequency Scale Factors. J. Phys. Chem. A 2007, 111, 11683-11700.

(27) Wong, M. W. Vibrational frequency prediction using density functional theory. Chem. Phys. Lett. 1996, 256, 391-399.

(28) Furuhashi, M.; Yoshinobu, J. Charge Transfer and Molecular Orientation of Tetrafluoro-tetracyanoquinodimethane on a HydrogenTerminated Si(111) Surface Prepared by a Wet Chemical Method. J. Phys. Chem. Lett. 2010, 1, 1655-1659.
(29) Pingel, P.; Zhu, L.; Park, K. S.; Vogel, J.-O.; Janietz, S.; Kim, E.G.; Rabe, J. P.; Brédas, J.-L.; Koch, N. Charge-Transfer Localization in Molecularly Doped Thiophene-Based Donor Polymers. J. Phys. Chem. Lett. 2010, 1, 2037-2041.

(30) Meneghetti, M.; Pecile, C. Charge-transfer organic crystals: Molecular vibrations and spectroscopic effects of electron-molecular vibration coupling of the strong electron acceptor TCNQF4. J. Chem. Phys. 1986, 84, 4149-4162.

(31) Zhang, S. B.; Wei, S.-H.; Zunger, A. Intrinsic n-type versus p-type doping asymmetry and the defect physics of ZnO. Phys. Rev. B 2001, 63, 075205 .

(32) Ellmer, K.; Bikowski, A. Intrinsic and extrinsic doping of $\mathrm{ZnO}$ and $\mathrm{ZnO}$ alloys. J. Phys. D: Appl. Phys. 2016, 49, 413002.

(33) Duva, G.; Pithan, L.; Zeiser, C.; Reisz, B.; Dieterle, J.; Hofferberth, B.; Beyer, P.; Bogula, L.; Opitz, A.; Kowarik, S.; Hinderhofer, A.; Gerlach, A.; Schreiber, F. Thin-Film Texture and Optical Properties of Donor/Acceptor Complexes. Diindenoperylene/ F6TCNNQ vs Alpha-Sexithiophene/F6TCNNQ. J. Phys. Chem. C 2018, 122, 18705-18714.

(34) The degree of charge transfer to the adsorbed molecules can be estimated from the $\nu(\mathrm{C}=\mathrm{C})$ vibrational frequency using a scheme proposed by Katayama et al. ${ }^{17}$ and Matsuzaki et al. [Sol. State Commun. 1980, 33 (4), 403-405]. For the estimate, we used the band at 1552 $\mathrm{cm}^{-1}$, which exhibits the strongest red shift $\left(45 \mathrm{~cm}^{-1}\right)$. Using the empiric formula proposed by Matsutzaki et al. (assuming that a red shift of $60 \mathrm{~cm}^{-1}$ corresponds to the formal charge of 1 ), we obtain a charge transfer of $0.75 \mathrm{e} / \mathrm{molecule}$. This value is very close to the value obtained by Katayama for $\mathrm{F}_{4}$-TCNQ. However, we would like to point out that the integer charge transfer model was used in our discussionand that the situation for a molecule adsorbed on a metal (considered in the work by Katayama et al. ${ }^{17}$ ) is quite different from that adsorbed on a dielectric.

(35) Schultz, T.; Niederhausen, J.; Schlesinger, R.; Sadofev, S.; Koch, $\mathrm{N}$. Impact of surface states and bulk doping level on hybrid inorganic/ organic semiconductor interface energy levels. J. Appl. Phys. 2018, 123, 245501.

(36) Sau, J. D.; Neaton, J. B.; Choi, H. J.; Louie, S. G.; Cohen, M. L. Electronic Energy Levels of Weakly Coupled Nanostructures: C60Metal Interfaces. Phys. Rev. Lett. 2008, 101, 026804.

(37) Hofmann, O. T.; Rinke, P.; Scheffler, M.; Heimel, G. Integer versus Fractional Charge Transfer at $\mathrm{Metal}(/$ Insulator)/Organic Interfaces: $\mathrm{Cu}(/ \mathrm{NaCl}) / \mathrm{TCNE}$. ACS Nano 2015, 9, 5391-5404. 